\title{
Disappearance of Integer Quantum Hall Effect
}

\author{
D. N. Sheng and Z. Y. Weng \\ Texas Center for Superconductivity and Department of Physics, University of Houston, Houston, Texas 77204-5506
}

(Received 28 August 1996)

\begin{abstract}
The disappearance of integer quantum Hall effect (IQHE) at strong disorder and weak magnetic field is studied in a lattice model. A generic sequence by which the IQHE plateaus disappear is revealed: higher IQHE plateaus always vanish earlier than lower ones, and extended levels between those plateaus do not float up in energy but keep merging together after the destruction of plateaus. All of these features remain to be true in the weak-field limit as shown by the thermodynamic localizationlength calculation. Topological characterization in terms of Chern integers provides a simple physical explanation and suggests a qualitative difference between the lattice and continuum models. [S0031-9007(96)02207-7]
\end{abstract}

PACS numbers: $73.40 . \mathrm{Hm}, 71.30 .+\mathrm{h}, 73.20 . \mathrm{Jc}$

It is an important issue how the integer quantum Hall effect vanishes in the weak magnetic-field limit. Several years ago, Khmel'nitzkii [1] and Laughlin [2] both argued that extended states at the centers of Landau levels would not disappear discontinuously or merge together, but should rather float up indefinitely towards higher energy to pass the Fermi level. This argument is also a key scenario in the global phase diagram proposed by Kivelson, Lee, and Zhang [3] for the quantum Hall effect. As a consequence, a direct transition from a higher IQHE state to an insulator state is forbidden [3]. Recently such a floating picture for extended states has been challenged by a numerical study by Liu et al. [4] based on a tight binding model (TBM), in which extended levels as represented by peaks of a finite-size localization length are found to disappear without changing their position at strong disorder. It indicates that the transition of IQHE states to an insulator occurs without involving a floating up of extended states. However, Yang and Bhatt [5] recently argued that this feature in a lattice model may not be contradictory to the conventional floating picture in the continuum limit. The argument goes as follows. They observed that in the TBM the critical strength $W_{c}$ of disorder by which all extended states vanish is roughly $W_{c} \sim 6 t$ ( $t$ is the hopping integral) at different magnetic flux strengths. If such a finite $W_{c}$ indeed persists into the weak magnetic-field case, a weaker disorder strength $\left(W<W_{c}\right.$ ) will not be able to destroy extended states and the only way to get into an insulator phase in the weakfield limit would be a floating up of extended levels as suggested by Khmel'nitzkii and Laughlin.

As these numerical calculations based on the TBM are all performed in relatively strong magnetic fields, one needs more conclusive evidences in order to resolve the above controversy and make the case relevant to the weak-field limit, which is of experimental interest. In this paper, we reexamine this problem in both strong and weak magnetic fields and present convincing results with regard to the physical mechanism of the disappearance of the
IQHE and its behavior in the weak-field limit. First, we show a systematic destruction of the IQHE at strong disorder, in which the IQHE plateaus are found to be destroyed in a one-by-one order from high to low energies. During such a process, extended levels separating different IQHE plateaus merge together and then disappear without floating up in energy, and the lowest extended level is the last to vanish at a critical disorder strength $W_{c}$. Such a picture for destroying the IQHE is shown to persist into the weak-field limit, where $W_{c}$ is found to decrease continuously with the decrease of magnetic fields, and can be extrapolated to zero at zero field limit, contrary to a fieldindependent $W_{c}$ as suggested in Refs. [5,6]. A topological reason for such a destruction of the IQHE by disorder is given based on the Chern number which characterizes extended states [7]: nonzero Chern integers with opposite sign are moving down from the band center to annihilate those in the lower-energy extended levels, and eventually a total annihilation of Chern numbers leads to a global insulating phase. In contrast to the finite-size localizationlength calculation in Ref. [4], though, we find that neighboring extended levels do merge together before their disappearance, as the result of moving and annihilation of Chern numbers.

The TBM Hamiltonian is given as follows:

$$
H=-\sum_{\langle i j\rangle} e^{i a_{i j}} c_{i}^{\dagger} c_{j}+\text { H.c. }+\sum_{i} w_{i} c_{i}^{\dagger} c_{i},
$$

where the hopping integral $t$ is taken as the unit, and $c_{i}^{\dagger}$ is a fermionic creation operator with $\langle i j\rangle$ referring to two nearest neighboring sites. A uniform magnetic flux per plaquette is given as $\phi=\sum_{\square} a_{i j}=2 \pi / M$, where the summation runs over four links around a plaquette. And $w_{i}$ is a random potential with strength $\left|w_{i}\right| \leq W / 2$ (note that the disorder strength $W$ here is twice bigger than that defined in Ref. [5]), and the white noise limit is considered with no correlation among different sites for $w_{i}$.

An overall picture for the Hall conductance calculated by the Kubo formula is shown in Fig. 1 with the flux 
strength $\phi=2 \pi / 8$ (only $E<0$ part is shown here). At weaker disorder $(W=1)$, three well-defined IQHE plateaus at $\sigma_{H}=\nu e^{2} / h(\nu=1,2,3)$ are clearly shown, corresponding to four Landau levels centered at the jumps of the Hall conductance at $E \leq 0$. With $W$ increasing from 1 to 6 , one sees a systematic destruction of these IQHE plateaus. For instance, at $W=2$ the $\nu=3$ plateau is the first beginning to disappear, while at $W=3$ and 4 the $\nu=2$ IQHE plateau starts to vanish too. Meantime the lowest plateau still remains well defined at $W=4$ and, in the inset of Fig. 1, such a $\nu=1$ IQHE plateau region is partially shown at different sample sizes, where all the curves cross at a fixed point corresponding to the Hall conductance $\frac{1}{2} e^{2} / h$ which is to be extrapolated to a sharp step of jump in the thermodynamic limit. By contrast, in those higher energy regions without the plateaus the Hall conductance is seen to monotonically decrease at larger lattice sizes. A breakdown of the lowest IQHE plateau is eventually found at larger $W$ 's ( $W=6$ case is shown in Fig. 1). Hall conductances at weaker flux strengths with $M=11,16$, and 24 all exhibit the similar generic features as in Fig. 1.

A key thing to understand the above evolution of IQHE plateaus is the localization-delocalization transition. It is a well-known fact that in the IQHE an extended state can be characterized by a topological index, namely, a nonzero Chern integer [7]. And the boundary-conditionaveraged Hall conductance is a summation of all those Chern numbers carried by states below Fermi surface [7,8]: $\left\langle\sigma_{H}\left(E_{f}\right)\right\rangle=e^{2} / h \sum_{\varepsilon_{m}<E_{f}} C^{(m)}$, where the Chern number $C^{(m)}$ is always an integer. (Numerically we have checked at $8 \times 8$ and $16 \times 16$ lattice sizes that $\left\langle\sigma_{H}\right\rangle$ coincides well with the Hall conductance in Fig. 1 obtained with the periodical boundary condition. There is a topological reason [9] why the quantization of Hall conduc-

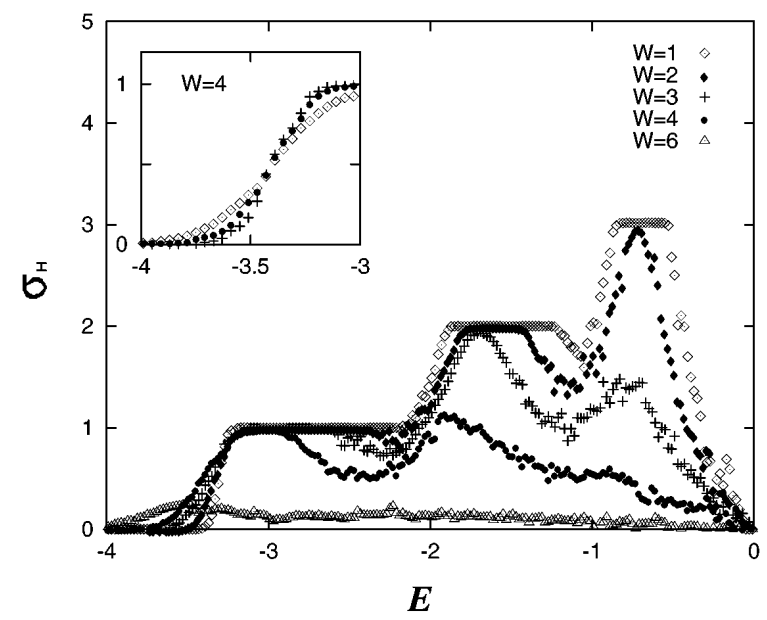

FIG. 1. The Hall conductance $\sigma_{H}$ as a function of energy $E$ is plotted for different disorder strength $W^{\prime}$ 's, at a lattice size $16 \times 16$. The inset shows the evolution of the $\nu=1 \mathrm{IQHE}$ plateau at $W=4$ with lattice size varying form $8 \times 8(\diamond)$, $16 \times 16(\bullet)$, to $24 \times 24(+)$. tance is not sensitive to the boundary condition.) Since its distribution in the thermodynamic limit decides delocalization regions, one may define states with nonzero Chern integers as extended states and calculate the corresponding density of extended states $\rho_{\text {ext }}[8]$. The results are presented in Fig. 2 with the same flux strength as in Fig. 1. At $W=1$ (weak disorder), well-defined peaks of $\rho_{\text {ext }}$ are located at centers of Landau-level bands, separated by localized regions represented by plateaus in Fig. 1. Widths of these peaks will approach zero in the thermodynamic limit [8]. Total Chern number for each of three lower-energy peaks is found to be exactly +1 , which is the reason leading to three quantized Hall plateaus at $+1,+2$, and +3 in units of $e^{2} / h$ shown in Fig. 1, when the Fermi surface is located between these peaks. The last peak closest to the band center carries a total Chern number -3 , which guarantees that the Hall conductance in Fig. 1 falls back to zero beyond the third plateau when the Fermi energy approaches $E=0$. This is a peculiar feature of a lattice model since, when the whole band is half-filled, the Hall conductance has to be zero. These Chern integers are conserved as long as they are well separated by localization regions, just like the quantization of the plateaus themselves.

The distribution of Chern integers (thus the extended states) begins to qualitatively change at stronger disorders. At $W=2$ and 3, two higher-energy peaks in Fig. 2 start to merge due to disorder scattering. Because these two peaks carry total Chern numbers of +1 and -3 , respectively, a mixing and annihilation of these Chern numbers results in a disappearance of the third plateau in Fig. 1, and leads to a substantial reduction of the magnitude of the Hall conductance in that region. By contrast, the lowest-energy peak remains separated from the rest spectrum up to $W=5$. In the inset of Fig. 2,

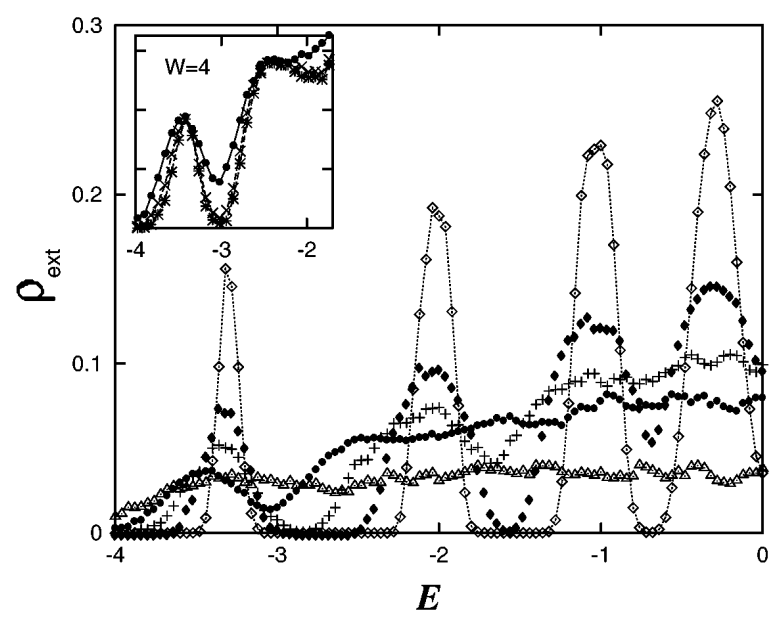

FIG. 2. The density of extended states $\rho_{\text {ext }}$ versus $E$. Disorder strength $W$ 's are the same as in Fig. 1 with a lattice size $8 \times 8$ (about 400 random-potential configurations are used). The inset shows $\rho_{\text {ext }}$ at $W=4$ with lattice sizes varying as $8 \times 8(\bullet), 16 \times 16(\times)$, and $24 \times 24(*)$. 
such a peak at $W=4$ as located at the energy $E_{c 1} \simeq$ -3.4 is shown to become narrower and sharper with the increase of lattice sizes, which indicates that a welldefined mobility edge still exists at $E_{c 1}$. Such a peak disappears finally as the negative Chern numbers reach to the region around $E_{c 1}$ as shown at $W=6$, and we find a monotonic decrease of $\rho_{\text {ext }}$ with larger lattice sizes which corresponds to a localization in the whole region. Therefore, the destruction of the IQHE at strong magnetic fields in the TBM can be well understood physically as due to a moving down of negative Chern integers from band center, caused by strong disorders, which mix with the positive Chern integers located at lower-energy extended levels and eventually annihilate them, leading to the insulator transition of the whole system.

Two questions remain to be answered here: First, since only a finite-size analysis has been done so far, one may wonder if the above picture of the insulator transition is still to be true in the thermodynamic limit, and second, whether such a picture also persists continuously into a weak magnetic-field case which is more relevant to the experimental situation. To answer the first question, we need to determine the thermodynamic localization length by a standard finite-size scaling method [10,11]. The results for the same field strength as in Figs. 1 and 2 are plotted in Fig. 3 for the case of $W=4$ and 5. At $W=4$, the thermodynamic localization length follows a scaling behavior $\xi \sim\left|E-E_{c 1}\right|^{-x}$ with the exponent $x=$ $2.4 \pm 0.1$ on the two sides of the critical energy $E_{c 1}$, which verifies the existence of a delocalization fixed point shown in the inset of Fig. 1 (the extended-state position $E_{c 1}=$ -3.40 also coincides with the one previously determined by the Chern number calculations). The localization length $\xi$ exhibits another divergence near $E_{c 2} \simeq-2.52$ ( $\rho_{\text {ext }}$ in the inset of Fig. 2 also shows a second peak here). Above $E_{c 2}$ one finds the localization length to be always finite up to the band center, indicating a localized region with zero Hall conductance as the negative Chern integers move down to $E_{c 2}$. Thus, between $E_{c 1}$ and $E_{c 2}$ there must remain a Hall conductance plateau as shown in Fig. 1. With $W$ increasing from 4 to $5, E_{c 1}$ is only slightly reduced (from -3.40 to -3.48 ), whereas $E_{c 2}$ moves down much more quickly (from -2.52 to -3.0 ), such that the $\nu=1$ IQHE plateau between $E_{c 1}$ and $E_{c 2}$ becomes narrower, and eventually these last two extended levels merge and disappear at a critical $W_{c}$. Correspondingly the $\nu=1$ plateau vanishes in Fig. 1 beyond this point. Thus, in contrast to the finite-size localization-length calculations in Ref. [4] where all the peaks of the localization length remain unchanged before their disappearance, the present thermodynamic localization-length data clearly indicate a moving down and mixing of extended levels before they disappear, consistent with the Chern number interpretation for the transition.

The magnetic flux strength $\phi=2 \pi / M$ at $M=8-24$ used in the previous calculations are relatively high

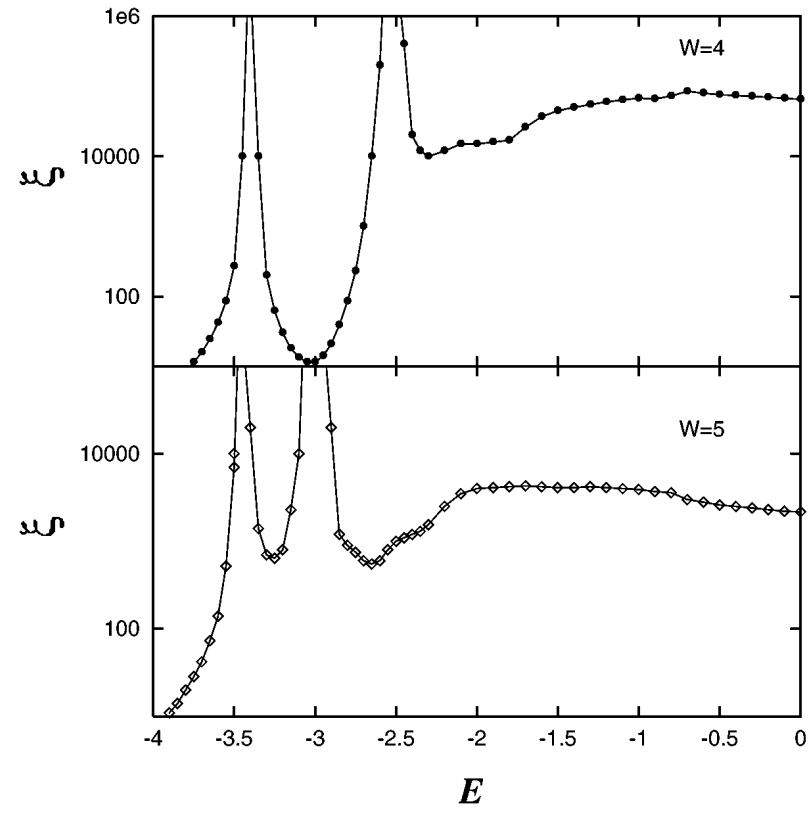

FIG. 3. Thermodynamic localization length $\xi$ is shown as a function of energy $E$ at $W=4$ and 5, respectively. Critical behaviors near divergent points are fit by scaling laws (see text).

fields. In order to answer the second question about the weak-field limit, we have to reduce the field strength substantially. In the following we inspect this case by examining the critical disorder $W_{c}$. Note that by increasing $W$, we always find that higher-energy extended levels disappear first, while the lowest one vanishes in the last without floating up in energy (which actually slightly shifts downwards). Then by following the evolution of the lowest extended level which can be determined from the thermodynamic localization length, one can decide a critical $W_{c}$, beyond which no extended states exist in the whole energy regime. $W_{c}$ 's obtained in this way are shown in Fig. 4 versus $1 / M$, the magnetic flux strength in units of $2 \pi$. We have been able to reach a weak field as low as $\phi=2 \pi / 384$ or $M=384$, and Fig. 4 shows that $W_{c}$ monotonically decreases with the magnetic-field strength which is extrapolated to zero at zero magnetic field. This result is at variance with the conjecture made by Yang and Bhatt [5] about roughly a constant $W_{c}(\sim 6)$ at $M \rightarrow \infty$ as extrapolated from the data within $M<13$. On the contrary, $W_{c}$ in Fig. 4 is well fit by a $1 / \sqrt{M}$ law at $M \geq 16$ which in turn means $W_{c} \propto \sqrt{\omega_{c}}\left(\omega_{c}\right.$ is the magnetic cyclotron energy and all quantities here are dimensionless as scaled by $t$ which is taken as the unit). If one estimates the Landau level broadening at weak field as $\Gamma \sim W^{2} / t$ [4], then $\Gamma \propto \omega_{c}$ is found at the critical $W_{c}$. It implies that once the neighboring Landau levels are coupled together due to the disorder scattering, the negative Chern integers near the band center will be able to flow all the way down to the band bottom and lead to the insulator transition. In other words, it 


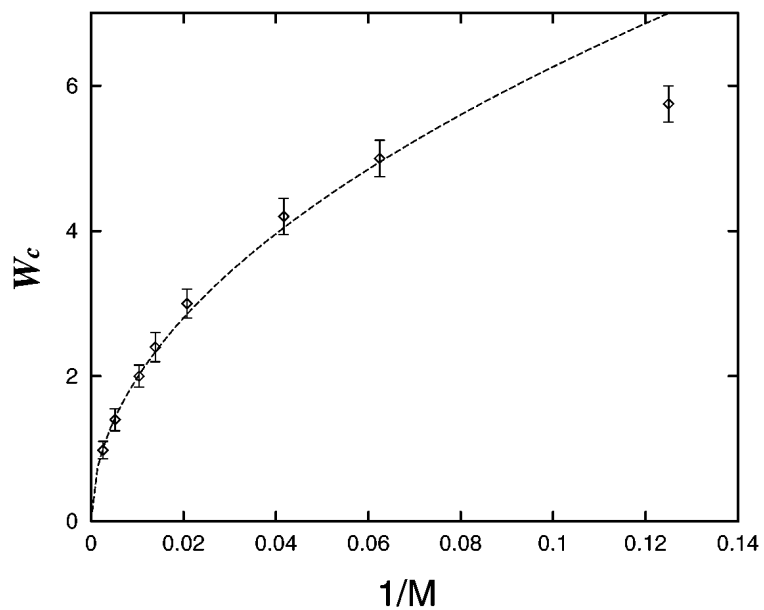

FIG. 4. The critical strength of disorder $W_{c}$ versus the magnetic flux strength (in unit $2 \pi$ ) $1 / M$. The dashed curve of a $1 / \sqrt{M}$ law well fits the data at $M \geq 16$.

is not necessary to have a strong $W_{c}$ comparable to the bandwidth in order to mix all the positive and negative Chern numbers. This is significant because the weak-field limit in real materials corresponds to the case that the Landau level spacing is much less than the bandwidth, and our results reveal that even in this limit the lattice effect (due to which one gets negative Chern numbers near the band center) is still crucial as far as the metal-insulator transitions are concerned. It suggests that the continuum model is oversimplified in describing such phenomena in real systems where the lattice structure is always present.

Finally, we would like to make several remarks. First, a limited floating-up picture can still be seen if the Landaulevel filling number is fixed. The reason is that the number of localized states below, say, the lowest extended level at $E_{c 1}$ can be enhanced by stronger disorders, so that when the filling number is fixed the Fermi level actually moves down or, relatively, the extended level shifts up, as already pointed out by Yang and Bhatt [5]. But such a relative "floating up" for the extended levels before their destruction is very limited and only within an order of one Landau level spacing, certainly not infinite, for the lowestenergy extended level as checked by us up to $M=192$. Second, the present results suggest that a direct transition from higher IQHE to an insulator becomes possible, after the IQHE plateau structure in Fig. 1 collapses from high energy downwards to the Fermi surface regime at a sufficient disorder, in contrast to the prediction from the global phase diagram. We shall leave discussions about comparison with experimental measurements [12] elsewhere. Last, a further theoretical understanding is still needed about why the negative Chern numbers near the band center can so easily move down to the band bottom once the neighboring Landau levels start to mix by disorder scattering.

The authors would like to thank T. Xiang, X. C. Xie, K. Yang, and C.S. Ting for stimulating and helpful discussions. The present work is supported by TCSUH, and TARP under Grant No. 3652182, and a grant from Robert Welch foundation.

[1] D. E. Khmel'nitzkii, Phys. Lett. 106A, 182 (1984).

[2] R. B. Laughlin, Phys. Rev. Lett. 52, 2304 (1984).

[3] S. Kivelson, D. H. Lee, and S. C. Zhang, Phys. Rev. B 46, 2223 (1992).

[4] D. Z. Liu, X. C. Xie, and Q. Niu, Phys. Rev. Lett. 76, 975 (1996).

[5] K. Yang and R. N. Bhatt, Phys. Rev. Lett. 76, 1316 (1996).

[6] T. Ando, Phys. Rev. B 40, 5325 (1989).

[7] D. J. Thouless et al., Phys. Rev. Lett. 49, 405 (1982); Q. Niu et al., Phys. Rev. B 31, 3372 (1985); D. P. Arovas et al., Phys. Rev. Lett. 60, 619 (1988).

[8] Y. Huo and R. N. Bhatt, Phys. Rev. Lett. 68, 1375 (1992).

[9] N. Imai et al., Phys. Rev. B 42, 10610 (1990); K. Ishikawa, Prog. Theor. Phys. Suppl. 107, 167 (1992); K. Ishikawa et al., Phys. Lett. A 210, 321 (1996).

[10] The method outlined in Ref. [11] is employed to determine the finite-size localization length based on strip lattices with the width $L$ varying from 16 to 80 and the length over $10^{5}$. Then the thermodynamic localization length $\xi$ can be extracted in terms of a standard oneparameter scaling analysis.

[11] A. MacKinnon and B. Kramer, Phys. Rev. Lett. 47, 1546 (1981); Z. Phys. B 53, 1 (1983).

[12] S. V. Kravchenko et al., Phys. Rev. Lett. 75, 910 (1995); D. Shahar et al., Phys. Rev. B 52, R14 372 (1995), and the references therein. 